\title{
Set-free Markov state model building
}

Marcus Weber, Konstantin Fackeldey, and Christof Schütte

Citation: The Journal of Chemical Physics 146, 124133 (2017); doi: 10.1063/1.4978501

View online: http://dx.doi.org/10.1063/1.4978501

View Table of Contents: http://aip.scitation.org/toc/jcp/146/12

Published by the American Institute of Physics

\section{Articles you may be interested in}

Microscopic derivation of particle-based coarse-grained dynamics: Exact expression for memory function The Journal of Chemical Physics 146, 124109124109 (2017); 10.1063/1.4978572

Calculating transition rates from durations of transition paths

The Journal of Chemical Physics 146, 124128124128 (2017); 10.1063/1.4979058

Wavelet Monte Carlo dynamics: A new algorithm for simulating the hydrodynamics of interacting Brownian particles

The Journal of Chemical Physics 146, 124111124111 (2017); 10.1063/1.4978808

Interaction entropy for protein-protein binding

The Journal of Chemical Physics 146, 124124124124 (2017); 10.1063/1.4978893

Defining and quantifying frustration in the energy landscape: Applications to atomic and molecular clusters, biomolecules, jammed and glassy systems

The Journal of Chemical Physics 146, 124103124103 (2017); 10.1063/1.4977794

How Markovian is exciton dynamics in purple bacteria?

The Journal of Chemical Physics 146, 124113124113 (2017); 10.1063/1.4978568

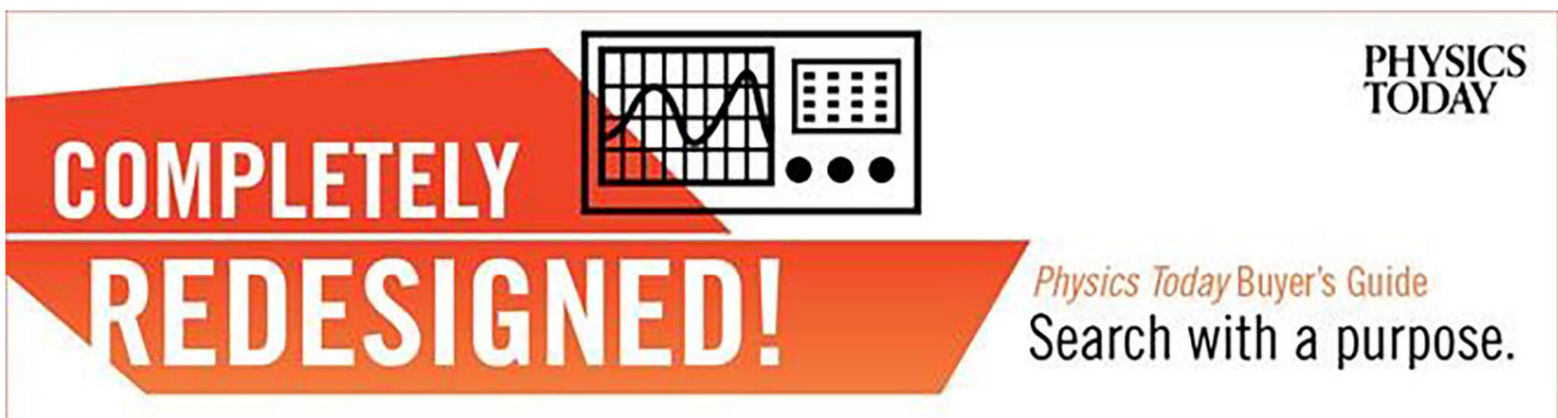




\title{
Set-free Markov state model building
}

\author{
Marcus Weber, ${ }^{1, a)}$ Konstantin Fackeldey, ${ }^{2, b)}$ and Christof Schütte ${ }^{3, c)}$ \\ ${ }^{1}$ Zuse Institute Berlin (ZIB), D-14195 Berlin, Germany \\ ${ }^{2} Z$ use Institute Berlin (ZIB) and Institute of Mathematics, Technical University Berlin, Berlin, Germany \\ ${ }^{3}$ Zuse Institute Berlin (ZIB) and Institute of Mathematics, Freie Universitaet Berlin, D-14195 Berlin, Germany
}

(Received 30 September 2016; accepted 1 March 2017; published online 31 March 2017)

\begin{abstract}
Molecular dynamics (MD) simulations face challenging problems since the time scales of interest often are much longer than what is possible to simulate; and even if sufficiently long simulations are possible the complex nature of the resulting simulation data makes interpretation difficult. Markov State Models (MSMs) help to overcome these problems by making experimentally relevant time scales accessible via coarse grained representations that also allow for convenient interpretation. However, standard set-based MSMs exhibit some caveats limiting their approximation quality and statistical significance. One of the main caveats results from the fact that typical MD trajectories repeatedly re-cross the boundary between the sets used to build the MSM which causes statistical bias in estimating the transition probabilities between these sets. In this article, we present a setfree approach to MSM building utilizing smooth overlapping ansatz functions instead of sets and an adaptive refinement approach. This kind of meshless discretization helps to overcome the recrossing problem and yields an adaptive refinement procedure that allows us to improve the quality of the model while exploring state space and inserting new ansatz functions into the MSM. Published by AIP Publishing. [http://dx.doi.org/10.1063/1.4978501]
\end{abstract}

\section{INTRODUCTION}

Molecular dynamics (MD) simulations are often used to estimate transition probabilities in phase space (or state space, respectively). In principle, one is interested in the following quantity:

$$
P(t, A, B)=\mathbb{P}_{\mu}\left(X_{t} \in B \mid X_{0} \in A\right) .
$$

In this formula, $A$ and $B$ are subsets of the state space. $P(t, A, B)$ denotes the conditional probability for a molecular process starting in $A$ to end up in $B$ after a certain time span $t$. This quantity is not meant for one single trajectory, it is an expectation value over all possible trajectories starting in $A$. Usually, $A$ and $B$ represent metastable conformations of the system, such that these transition probabilities are small and cannot be sampled efficiently by performing long-time trajectories of the molecular system. More precisely, estimating $P(t, A, B)$ based on molecular simulation of many trajectories starting in $A$ and rarely reaching $B$ in a given time $t$ is not a good idea. In this article we recall that (1) can be seen as a Galerkin discretization of a transfer operator $T_{t}$. The (time dominating) slowest processes of the molecular system are correlated with the highest eigenvalues and their eigenfunctions of $T_{t}$. Thus, finding dominating molecular processes can be turned into solving an eigenfunction problem of an (self-adjoint) operator $T_{t}$. The sampling problem turns into an eigenfunction problem.

Is this class of problems easier to solve? It is a good question, because the state space is high-dimensional and we try to

\footnotetext{
a)weber@zib.de

b) fackeldey@math.tu-berlin.de

c) Christof.Schuette@fu-berlin.de
}

solve a function approximation problem in this space. Furthermore, for a Galerkin discretization we need to compute inner products, which means we have to solve high-dimensional integrals. Both of these problems have been addressed in the literature. ${ }^{1}$ For the solution of a function approximation problem, we will propose an adaptive meshless discretization method. Whereas, for the quadrature problem we will apply a Monte-Carlo approach which will be based on biased stochastic MD simulations. There is good hope to overcome the sampling problem by this approach, because the approximated eigenfunctions have a rather simple structure (nearly piece-wise constant). The more rarely transitions between $A$ and $B$ occur, the easier the eigenfunctions become. On the other hand, there is no need for the simulation of trajectories which connect $A$ and $B$. For Monte-Carlo quadrature the trajectories are only needed for the evaluation of the (localized) inner products.

\section{THEORY}

\section{A. Molecular dynamics}

We consider a molecular system for which we would like to know transition probabilities (1) according to a given stochastic or deterministic molecular dynamics. Let $\mathbb{X}$ denote its phase space or state space, respectively, depending on the form of MD. The result of a MD simulation is a trajectory $\left(x_{t}\right)$, where $t$ runs through all discrete time steps of the simulation and $x_{t} \in \mathbb{X}$ for all $t$. Typically this trajectory results from (temporal) discretization of a set of equations of motion governed by the (potential) energy function $V$ that in principle have time-continuous trajectories $\left(X_{t}\right)_{t \in \mathbb{R}}$ as their solutions. For a canonical ensemble MD, one typically assumes that $\left(x_{t}\right)$ is (approximately) distributed according to the stationary 
distribution or invariant measure

$$
\mu(x)=\frac{1}{Z} \exp (-\beta V(x)),
$$

resulting from the ergodicity of $\left(X_{t}\right)$ which guarantees that for an appropriate macro-observable $f$ we have

$$
\lim _{T \rightarrow \infty} \frac{1}{T} \int f\left(X_{t}\right) d t=\int f(x) \mu(x) d x .
$$

When one performs very long MD simulations repeatedly starting from the same initial state, then the resulting trajectories differ. This may be caused by the chaotic properties of thermostatted $\mathrm{MD}^{2}$ for long enough time scales or it may result from the fact that the underlying equations of motion are stochastic as in the case of Langevin or Smoluchowski dynamics. For the following, it does not matter which of the two cases we consider; we will just assume that there is a probability distribution $p(t, x, y)$ for observing trajectories $\left(X_{t}\right)$ that start in $X_{0}=x$ and end in $X_{t}=y$. The transfer operator $T_{t}$ associated with the dynamics is defined by

$$
T_{t} f(x)=\mathbb{E}\left(f\left(X_{t}\right) \mid X_{0}=x\right)=\int f(y) p(t, x, y) d y,
$$

where the condition $X_{0}=x$ means that all trajectories start in $x$. The transfer operator tells us how the expectation value of an observable $f$ is evolving under the dynamics. Specific forms of the transfer operator, e.g., for thermostatted Hamiltonian dynamics, can be found in the literature. ${ }^{2,3}$

By introducing the scaled scalar product

$$
\langle f, g\rangle_{\mu}=\int f(x) g(x) \mu(x) d x,
$$

we can write equilibrium correlation functions as

$$
\begin{aligned}
\left\langle f\left(X_{t}\right) g\left(X_{0}\right)\right\rangle & =\mathbb{E}\left(f\left(X_{t}\right) g\left(X_{0}\right) \mid X_{0} \sim \mu\right) \\
& =\int g(x) T f(x) \mu(x) d x=\langle g, T f\rangle_{\mu} \\
& =\left\langle T^{*} f, g\right\rangle_{\mu},
\end{aligned}
$$

where $T^{*}$ denotes the adjoint transfer operator. Similarly, we find that the transition probability between sets $A$ and $B$ in time $t$ can be expressed via the transfer operator also,

$$
\begin{aligned}
P(t, A, B) & =\frac{1}{\mu(A)} \int_{A} \int_{B} p(t, x, y) \mu(x) d x d y \\
& =\frac{1}{\mu(A)}\left\langle\mathbf{1}_{A}, T \mathbf{1}_{B}\right\rangle_{\mu} \\
& =\frac{1}{\mu(A)}\left\langle\mathbf{1}_{B}\left(X_{t}\right) \mathbf{1}_{A}\left(X_{0}\right)\right\rangle,
\end{aligned}
$$

where $\mu(A)=\int_{A} \mu(x) d x=\left\langle\mathbf{1}_{\mathbb{X}}, \mathbf{1}_{A}\right\rangle_{\mu}$ and $\mathbf{1}_{A}$ denotes the characteristic function of set $A$, i.e., $\mathbf{1}_{A}(x)=1$ if $x \in A$ and $=0$ otherwise.

The dynamics is called reversible if $\left(X_{t}\right)$ satisfies the detailed balance condition $\mu(x) p(t, x, y)=\mu(y) p(t, y, x)$. If this is the case then the transfer operator is self-adjoint with respect to the scaled scalar product, i.e., we have $T_{t}=T_{t}^{*}$ such that all of its eigenvalues are real-valued. ${ }^{3,4}$ Then, since the largest (in modulus) eigenvalue of any transfer operator for ergodic dynamics is $\lambda=1$, the eigenvalues of $T_{\tau}$ for an arbitrary time $\tau>0$ can be ordered,

$$
1=\lambda_{1}>\lambda_{2} \geq \lambda_{3} \geq \ldots
$$

Let $u_{j}$ denote the eigenfunction of $T_{\tau}$ associated with $\lambda_{j}$,

$$
T_{\tau} u_{j}=\lambda_{j} u_{j} .
$$

Because of the self-adjointness of $T_{\tau}$, these eigenfunctions are orthogonal with respect to $\langle\cdot, \cdot\rangle_{\mu}{ }^{2}$ such that we find that ${ }^{5-7}$

$$
\left\langle f\left(X_{n \tau}\right) g\left(X_{0}\right)\right\rangle=\sum_{j=1}^{m} \lambda_{j}^{n}\left\langle g, u_{j}\right\rangle_{\mu}\left\langle u_{j}, f\right\rangle_{\mu} .
$$

That is, for large enough times (=large enough $n$ ) the largest eigenvalues of $T_{\tau}$ completely dominate the kinetic relaxations in the molecular system on associated dominant time scales $^{3,5}$

$$
t_{j}=-\frac{\tau}{\log \lambda_{j}}, \quad j>1
$$

with $t_{1}=\infty$ describing the asymptotic decay to the stationary distribution at infinite time. The observation that the dominant eigenvalues of the transfer operator describe the long time relaxation kinetics and transition probabilities of the molecular system can also be verified for non-reversible systems like Langevin dynamics. ${ }^{3}$

The easiest example for the equations of motion in molecular dynamics is diffusion in the energy landscape $V$, i.e.,

$$
\dot{x}_{t}=-\nabla_{x} V\left(x_{t}\right)+\sqrt{2 \beta^{-1}} \dot{B}_{t},
$$

where $B_{t}$ denotes Brownian motion. This kind of dynamics is just a simplistic example for reversible MD but will provide a means for illustration in the following. The dynamics given by (3) is reversible and ergodic with respect to $\mu^{3}$. In this case the transfer operator has the form ${ }^{4}$

$$
T_{t}=\exp (t L), \quad L=\beta^{-1} \Delta_{x}-\nabla_{x} V(x) \cdot \nabla_{x},
$$

where $\Delta_{x}$ denotes the Laplacian operator.

\section{B. Generalized eigenvalue problem}

The main idea of MSM building is to construct a (small) transition matrix whose leading eigenvalues and vectors are very good approximations of the dominant eigenvalues and functions of the transfer operator $T_{\tau}$ of the system for some preselected time scale $\tau$. Therefore, we have to consider the eigenvalue problem

$$
T u=\lambda u
$$

of the full transfer operator $T=T_{\tau}$ of the dynamics.

As a starting point for the discretization of the eigenvalue problem (4) we consider a set of $m$ non-negative ansatz functions $\Phi_{1}, \ldots, \Phi_{m}$ that form a partition of unity, i.e., for all states $x \in \mathbb{X}$ we have that

$$
\sum_{j=1}^{m} \Phi_{j}(x)=1 .
$$

Each ansatz function $\Phi_{j}$ represents the weight

$$
\hat{\mu}_{j}=\int \Phi_{j}(x) \mu(x) d x=\left\langle\Phi_{j}, \mathbf{1}\right\rangle_{\mu}>0,
$$

since the partition of unity property guarantees that $\sum_{j} \hat{\mu}_{j}=1$. 
All functions that can be expressed as linear combinations of these ansatz functions form the linear subspace

$$
D=\left\{u: \quad u=\sum_{j=1}^{m} \bar{u}_{j} \Phi_{j}, \bar{u}_{j} \in \mathbb{R}\right\} .
$$

Based on the ansatz space $D$, Galerkin discretization of the eigenvalue problem (4) assumes ${ }^{3,8}$

$$
u=\sum_{j=1}^{m} \bar{u}_{j} \Phi_{j}, \quad \text { i.e., } \quad \sum_{j=1}^{m} \bar{u}_{j}\left(T \Phi_{j}-\lambda \Phi_{j}\right)=0,
$$

then multiplies the last equation from the left with $\Phi_{k} / \hat{\mu}_{k}$, using the scalar product $\langle\cdot, \cdot\rangle_{\mu}$, which results in

$$
\sum_{j=1}^{m} \frac{1}{\hat{\mu}_{k}}\left(\left\langle\Phi_{k}, T \Phi_{j}\right\rangle_{\mu}-\lambda\left\langle\Phi_{k}, \Phi_{j}\right\rangle_{\mu}\right) \bar{u}_{j}=0 .
$$

Introducing the two $m \times m$ matrices $P$ and $M$ with entries

$$
P_{k j}=\frac{1}{\hat{\mu}_{k}}\left\langle\Phi_{k}, T \Phi_{j}\right\rangle_{\mu}, \quad M_{k j}=\frac{1}{\hat{\mu}_{k}}\left\langle\Phi_{k}, \Phi_{j}\right\rangle_{\mu},
$$

and the coefficient vector $\bar{u}=\left(\bar{u}_{j}\right)_{j=1, \ldots, m}$, we can write Equation (5) in the form of a generalized eigenvalue problem, ${ }^{3,9}$

$$
P \bar{u}=\lambda M \bar{u} .
$$

It has been shown ${ }^{3,8}$ that the solution of this generalized eigenvalue problem is the best possible approximation of the solution of the full eigenvalue problem (4) if restricted to the ansatz space $D . P$ is a stochastic matrix with stationary distribution $\hat{\mu}=\left(\hat{\mu}_{1}, \ldots, \hat{\mu}_{m}\right)$. In fact it can be shown that the discretized eigenvalue problem (6) inherits most of the structural properties of (4), e.g., no eigenvalue has absolute value larger than 1 and all its eigenvalues are real-valued if the dynamics is reversible. $^{3}$

\section{Set-based MSMs}

The most prominent example for a partition of unity results from set complete partition of state/phase space: Assume that the disjoint sets $A_{1}, \ldots, A_{m}$ decompose state space, i.e., $\cup_{j} A_{j}=\mathbb{X}$. Then their characteristic functions $\Phi_{j}=\mathbf{1}_{A_{j}}$ form a partition of unity and we find that ${ }^{3}$

$$
P_{k j}=P\left(\tau, A_{k}, A_{j}\right)
$$

and $M_{k j}=\delta_{k j}$, the standard identities used in set-based MSM building. In this case, MSM building reduces to (A) computing the transition matrix $P$ and (B) solving the eigenvalue problem $P \bar{u}=\lambda \bar{u}$ for the dominant eigenvalues and eigenvectors. Since the entries of $P$ are just the transition probabilities between the discretization sets $A_{j}$, it can be seen easily that the computation of $P_{k j}$ only requires a couple of trajectories of length $\tau$ starting in $A_{k}$ and setting $P_{k j}$ to the fraction of these trajectories that ends up in $A_{j}$. This procedure has been studied to quite some extent $\mathrm{t}^{2-4,9,10}$ and has been applied to a variety of molecular systems. Its main disadvantage is that a reduction of the error of the discretization often requires the unwanted refinement of sets in the transition region between the metastable sets which can lead to an explosion of the number of sets needed. ${ }^{11}$

Therefore, alternative forms of set-based MSM building do not use complete set partitions of state space but require some disjoint core sets or milestones only that have to be placed at the attractive core of the main metastable sets. ${ }^{9}$ Then the partition of unity is formed by the committor functions associated with these core sets. Again, the computation of the discretization matrices $P$ and $M$ can be reduced to transition counts based on trajectories. ${ }^{3,12}$ However, depending on the size and location of the core sets, quite long trajectories may be needed which can create severe limitations. ${ }^{12}$

\section{Set-free MSMs}

The form of partition of unity that we will discuss next is constructed using radial basis functions: Based on a collection of $m$ so-called base points $q_{j} \in \mathbb{X}$, we define

$$
\Phi_{j}(x)=\gamma(x) \exp \left(-\alpha_{j} d\left(x-q_{j}\right)^{2}\right),
$$

where $\alpha_{j}$ is a positive constant, $d$ a distance measure ( $d$-proximity), for example, the Euclidean measure $d(x)^{2}$ $=\sum_{k=1}^{n} x_{j}^{2}$, and $\gamma$ is chosen so that the partition of unity condition is satisfied, i.e.,

$$
\gamma(x)^{-1}=\sum_{j=1}^{m} \exp \left(-\alpha_{j} d\left(x-q_{j}\right)^{2}\right) .
$$

The efficient computation of the discretization matrices $P$ and $M$ based on the ansatz space $D$ associated with a radial basis function based on partition of unity has rarely been discussed ${ }^{1}$ up to now and will form the main part of Sec. III.

\section{E. Discretization error}

In order to estimate the discretization error of the eigenvalue problem, we first have to understand that the Galerkin discretization can be understood as an orthogonal projection $Q$ (orthogonal with respect to $\langle\cdot, \cdot\rangle_{\mu}$ ) from the full function space to the ansatz space $D$,

$$
Q v=\sum_{k, j=1}^{n}\left(M^{-1}\right)_{k j}\left\langle\Phi_{k}, v\right\rangle_{\mu} \Phi_{j} .
$$

Let the dominant eigenvalues of the full transfer operator $T=T_{\tau}$ be denoted

$$
1=\lambda_{1}>\lambda_{2} \geq \ldots \geq \lambda_{m},
$$

with associated eigenfunctions $u_{j}, j=1, \ldots, m$. Moreover, let the leading eigenvalues of the generalized eigenvalue problem (6) be denoted

$$
1=\hat{\lambda}_{1}>\hat{\lambda}_{2} \geq \ldots \geq \hat{\lambda}_{m}
$$

Then the discretization error is characterized by

$$
\max _{j=2, \ldots, m}\left|\lambda_{j}-\hat{\lambda}_{j}\right| \leq 2 \lambda_{j} \delta,
$$

where $\delta$ denotes the projection error of the leading eigenvectors to $D$,

$$
\delta=\max _{j=1, \ldots, m}\left\|u_{j}-Q u_{j}\right\|,
$$

where $\|v\|=\sqrt{\langle v, v\rangle_{\mu}}$. 


\section{METHODS}

In this section, we will first construct efficient methods for computing the entries of the discretization matrices $P$ and $M$ based on radial basis ansatz functions of the form (7). Second, we will present an algorithm for refining a discretization by adding ansatz functions in order to decrease the error of the discretization.

\section{A. Computation of $\boldsymbol{P}$ and $\boldsymbol{M}$}

We first have to discuss how to compute scalar products of the form

$$
\left\langle\Phi_{k}, u\right\rangle_{\mu}=\int \Phi_{k}(x) u(x) \mu(x) d x,
$$

for an arbitrary function $u$ that we will specify later. Since $\mu(x)=\exp (-\beta V(x)) / Z$ we have

$$
\left\langle\Phi_{k}, u\right\rangle_{\mu}=\int \frac{\gamma(x)}{Z} u(x) \exp \left(-\beta U_{k}(x)\right) d x,
$$

with the energy function

$$
U_{k}(x)=V(x)+\frac{\alpha_{k}}{\beta} d\left(x-q_{k}\right)^{2},
$$

which is composed out of the original energy function plus an extra harmonic potential. Molecular dynamics simulation runs with the potential $U_{k}$ instead of $V$ are easily implemented in all standard MD codes and will sample the invariant measure

$$
\begin{aligned}
& \mu_{k}(x)=\frac{1}{Z_{k}} \exp \left(-\beta U_{k}(x)\right), \\
& Z_{k}=\int \exp \left(-\beta U_{k}(x)\right) d x
\end{aligned}
$$

so that

$$
\left\langle\Phi_{k}, u\right\rangle_{\mu}=\frac{Z_{k}}{Z} \int \gamma(x) u(x) \mu_{k}(x) d x .
$$

When performing MD simulations with the energy function $U_{k}$, we will produce sample points $\left(x_{1, k}, \ldots, x_{L, k}\right)$ that are distributed according to $\mu_{k}$. For each potential $U_{k}$ this set of points will be referred to as the restraint sampling points in the following. We remark that for the procedure which generates the restraint sampling points, any rapidly mixing sampling scheme can be applied independent of the choice of the desired $T_{t}$-dynamics. The reason is that these sampling points only act as quadrature points for a Monte Carlo evaluation of the inner products. In the numerical examples, therefore, a standard Metropolis Hastings (MC) sampling method will be applied.

Using the restraint sampling points, the above integral can be approximated by the respective mean value

$$
\left\langle\Phi_{k}, u\right\rangle_{\mu} \approx \frac{Z_{k}}{Z} \frac{1}{L} \sum_{i=1}^{L} \gamma\left(x_{i, k}\right) u\left(x_{i, k}\right),
$$

where the error will vanish for $L \rightarrow \infty$. This result allows us to calculate the entries of the mass matrix $M$,

$$
M_{k j}=\frac{\left\langle\Phi_{k}, \Phi_{j}\right\rangle_{\mu}}{\left\langle\Phi_{k}, \mathbf{1}\right\rangle_{\mu}} \approx \tilde{M}_{k j}(L)=\frac{\sum_{i=1}^{L} \gamma\left(x_{i, k}\right) \Phi_{j}\left(x_{i, k}\right)}{\sum_{i=1}^{L} \gamma\left(x_{i, k}\right)}
$$

The entries of $P$, however, require knowledge regarding the action of $T$ on $\Phi_{j}$,

$$
P_{k j} \approx \frac{\sum_{i=1}^{L} \gamma\left(x_{i, k}\right) T \Phi_{j}\left(x_{i, k}\right)}{\sum_{i=1}^{L} \gamma\left(x_{i, k}\right)}
$$

In order to approximate $T \Phi_{j}\left(x_{i, k}\right)$, we need $K$ trajectories of time length $\tau$ of the original molecular dynamics with respect to the energy function $V$, all starting in $x_{i, k}$. Let $z_{i, k, l}$ denote the end point of the $l$-th of these trajectories. Then

$$
T \Phi_{j}\left(x_{i, k}\right)=\mathbb{E}\left(\Phi_{j}\left(X_{\tau}\right) \mid X_{0}=x_{i, k}\right) \approx \frac{1}{K} \sum_{l=1}^{K} \Phi_{j}\left(z_{i, k, l}\right),
$$

where, again, the error will vanish for $K \rightarrow \infty$. Putting things together, we can calculate the entries of $P$ via

$$
P_{k j} \approx \tilde{P}_{k j}(L, K)=\frac{1}{K} \frac{\sum_{i=1}^{L} \sum_{l=1}^{K} \gamma\left(x_{i, k}\right) \Phi_{j}\left(z_{i, k, l}\right)}{\sum_{i=1}^{L} \gamma\left(x_{i, k}\right)} .
$$

Conclusively we found the following algorithm for approximately computing the entries of $P_{k j}$ and $M_{k j}$, and approximate the dominant eigenvalues $\lambda$ and eigenfunctions $u$ of $T$ :

1. Assume that the ansatz functions are of the form (7). Select large enough $K$ and $L$.

2. For each $k=1, \ldots, m$ :

- Compute $L$ sampling points $x_{k, i}, i=1, \ldots, L$, using MD simulation with respect to the energy function $U_{k}$ given in (10).

- Compute $\tilde{M}_{k j}(L)$ for all $j=1, \ldots, m$ using (11).

- For each $i=1, \ldots, L$ : Compute $K$ trajectories of length $\tau$ with end points $z_{k, i, l}, l=1, \ldots, K$, using MD simulation with respect to the original energy function $V$.

- Compute $\tilde{P}_{k j}(L, K)$ for all $j=1, \ldots, m$ using (12).

3. Solve the generalized eigenvalue problem $\tilde{P} \bar{u}=\lambda \tilde{M} \bar{u}$.

\section{B. Finding metastable sets}

Finding the eigenvalues $\lambda_{i}$ of the transfer operator $T$ is only one part of the problem. The other part is identifying the sets in (1), such that the transition probabilities are small. We will use PCCA $+{ }^{13,14}$ in order to characterize the metastabilities of the dynamical system. PCCA+ is based on the following concept: A membership function $\chi: \mathbb{X} \rightarrow[0,1]$ represents a kind of "fuzzy"-set, it assigns a grade of membership $\chi(x)$ to every state $x \in \mathbb{X}$ of the system. If $T \chi \approx \chi$, then this set is metastable with regard to the dynamics encoded in the transfer operator $T$. If we try to find a set of $n$ membership functions $\chi_{1}, \ldots, \chi_{n}$ which are linearly independent and which are all as metastable as possible, then this can be written in terms of a variational principle and the functions $\chi$ are optimally given by a linear combination of the leading eigenfunctions of $T$. In the above algorithm we have already computed an approximation of these eigenfunctions. Given the $n$ leading generalized eigenvectors $\bar{u}_{1}, \ldots, \bar{u}_{n} \in \mathbb{R}^{m}$ with $\tilde{P} \bar{u}_{j}=\lambda \tilde{M} \bar{u}_{j}$ according to the above algorithm, we construct approximated membership functions $\tilde{\chi}_{1}, \ldots, \tilde{\chi}_{n}$ by the linear combination 
approach in the space of ansatz functions $\Phi$,

$$
\tilde{\chi}_{i}(x)=\sum_{j=1}^{m} c_{i j} \Phi_{j}(x) .
$$

If the vectors $c_{i}=\left(c_{i 1}, \ldots, c_{i m}\right)^{T}$ are non-negative and form a partition of unity, such that $\sum_{i=1}^{n} c_{i j}=1$, then $\tilde{\chi}$ is non-negative and forms a partition of unity, because it is a convex combination of the ansatz functions. In order to compute the vectors $c_{i}$, we denote them as a linear combination of the eigenvectors $\bar{u}_{j}$ via $c_{i k}=\sum_{j=1}^{n} a_{i j} \bar{u}_{j k}$. PCCA+ is used to find an optimal set of linear combination factors $a_{i j} .13,14$

\section{Constructing an initial discretization}

In the beginning of the meshless approach, an initial sampling of the state space $\mathbb{X}$ is needed. This sampling need not be trajectory-based, it even need not represent the invariant measure $\mu$. Additionally, it need not be completely covering $\mathbb{X}$. In the numerical example, we will start with a very localized sampling only having discovered one out of three metastable states of the system. Out of this initial sampling $s_{1}, \ldots, s_{I}$, we will pick a predefined number of initial base points. The picking algorithm is done in the following way. ${ }^{15}$

- In the first step an arbitrary base point $q_{1}$ is picked. Then the sampling point $q_{2}$ with the maximal distance to $q_{1}$ is picked.

- In the next steps, always the sampling point $q_{k}$ is picked which has the maximal minimal distance to all given base points $q_{1}, \ldots, q_{k-1}$. That is, $q_{k}$ is the point $s$ out of the set of sampling points $s_{1}, \ldots, s_{I}$ which maximizes the following expression: $\max _{s} \min _{j=1, \ldots, k-1 ; s \neq q_{j}} d\left(s-q_{j}\right)^{2}$.

- The last base point $q_{m}$ picked in this way has the maximal minimal distance $d_{\min }$ to all other base points.

The basis functions $\Phi_{k}$ are now almost constructed; finally we only need to define $\alpha_{k}$. The more dense the base points are located, the higher the $\alpha$-values should be, because the restraint simulations should be localized around their individual base points. In theorem 5.10 in the doctoral thesis, ${ }^{1}$ it is shown that the error $\|\chi-\tilde{\chi}\|_{L^{1}(\mu)}$ between the true membership functions $\chi$ and the best approximation $\tilde{\chi}$ (based on the meshless discretization) is small only if $\alpha$ is sufficiently large. In order to assure a small overlap between restraint samplings for base points having a greater distance than $2 d_{\min }$, the scalar factors $\alpha_{j}$ are (equally) chosen to be proportional to $d_{\min }^{-2}$ (formula (80) in the doctoral thesis ${ }^{1}$ ). For a predefined parameter $\sigma$, we define $\alpha_{j}=\sigma / d_{\text {min }}^{2}$ for $j=1, \ldots, m$. The question remains, how to sufficiently cover the relevant part of $\mathbb{X}$ with an initial discretization. One could apply the existing methods for a good initial sampling of $\mathbb{X}$ (ConCoord,${ }^{16}$ GLAT, ${ }^{17}$ taboo search, ${ }^{18}$ or continuation methods ${ }^{19}$ ). Alternatively, the above picking algorithm could be used (and will be used in the numerical example) to "fill" $\mathbb{X}$ : After we have constructed the basis functions $\Phi_{k}$, we perform the restraint simulations according to the penalty potentials $U_{k}$. A major challenge in molecular simulation is that the trajectories of the system run into the next closest minimum, which is also known as the trapping problem. This fact can hinder the system to explore additional parts of the state space $\mathbb{X}$. If we have picked a large number of base points, then $\alpha_{k}$ will be large and the penalty potentials diminish a trapping. We simply restart the picking algorithm on the basis of all sampling points stemming from the restraint simulations and pick again the same number $m$ of base points out of this set. If $d_{\min }$ has extended compared to the initial picking, then new parts of the state space have been found and we have to restart the restraint simulations and picking again. This iteration is done, until $d_{\min }$ does not extend anymore. The result of this procedure is our initial basis for the refinement steps.

\section{Solving the generalized eigenvalue problem and refinement}

The general eigenvalue problem (6) in general is illconditioned, especially, when solving it by inverting $\tilde{M}$. We, therefore, tread a different path by only computing the leading eigenvalues of $\tilde{P}$, i.e.,

$$
\tilde{P} \bar{u}_{P, i}=\lambda_{P, i} \bar{u}_{P, i} .
$$

This eigenvalue problem typically is well-conditioned. Using PCCA+ the coefficients $c_{j}, j=1, \ldots$, , n are computed as a linear combination of the leading eigenvectors of (13), i.e., $c_{i k}$ $=\sum_{j=1}^{n} a_{i j} \bar{u}_{P, j k}$, which we can also denote using the linear space spanned by the leading eigenvectors, $c_{j} \in \operatorname{span}\left(\left\{\bar{u}_{P, i}\right\}\right), j$ $=1, \ldots, n$. If

$$
\tilde{M} c_{j} \in \operatorname{span}\left(\left\{\bar{u}_{P, i}\right\}\right), \quad j=1, \ldots, n,
$$

then the eigen vectors $\left(\bar{u}_{i}\right)$ of (6) meet

$$
c_{j} \in \operatorname{span}\left(\left\{\bar{u}_{i}\right\}\right), \quad j=1, \ldots, n,
$$

which is equivalent to

$$
\tilde{M} c_{j}-\Pi_{\mu}^{\bar{u}_{j}}\left(\tilde{M} c_{j}\right)=0, \quad j=1, \ldots, n .
$$

In this equation $\Pi_{\mu}^{\bar{u}_{j}}$ is the $\mu$ weighted orthogonal projection onto the eigenvector $\bar{u}_{j}$ which in its matrix form can be denoted by $\Pi_{\mu}^{\bar{u}_{j}}=\bar{u} \bar{u}^{T} W$ with $W=\operatorname{diag}\left(\hat{\mu}_{k}\right)$. Summing up we have the following algorithm for solving the generalized eigenvalue problem (6):

1. Compute the $n$ leading eigenvectors $\left\{\bar{u}_{P, i}\right\}$ of (13).

2. Evaluate the coefficients $c_{j}, j=1, \ldots, n$ via PCCA+.

3. Compute for each basis function $\Phi_{k}, k=1, \ldots, m$ the error

$$
r_{k}=\sum_{i=1}^{n}\left|\left(\tilde{M} c_{i}-\Pi_{\mu}^{\bar{u}_{j}}\left(\tilde{M} c_{i}\right)\right)_{k}\right| .
$$

4. For some given tolerance TOL,

$$
\begin{aligned}
& r_{k}<\text { TOL break, } \\
& r_{k} \geq \text { TOL add new basis function }(m \rightarrow m+1) \\
& \quad \text { and go to } 1 .
\end{aligned}
$$

We remark that (14) can be interpreted as a condition on the discretization, which guarantees that solving the generalized eigenvalue problem (6) is not ill-conditioned. More precisely if (14) holds, then the eigenvalues of (6) are given by $\lambda_{i}=\lambda_{P, i} / \lambda_{M, i}$ where $\lambda_{P, i}$ are the eigenvalues of $\tilde{P}$ and $\lambda_{M, i}$ are 
the eigenvalues of $\tilde{M}$, respectively. The algorithm can also be considered as an adaptive refinement strategy with indicator $r_{k}$. Within each basis function $\Phi_{k}, k=1, \ldots, m$, we check if the restraint sampling points $\left(x_{1, k}, \ldots, x_{L, k}\right)$ are distributed according to the local invariant measure $\mu_{k}$. For this purpose we apply the Gelman-Rubin ${ }^{20}$ criterion as described in the literature. ${ }^{21}$ One further refinement strategy ${ }^{1}$ had been proposed based on a perturbation of the shape parameter $\alpha$. Common to all refinement strategies is that they require the addition of new basis functions, which can be done in an efficient way as described in Sec. III E.

\section{E. Adding new base points}

In order to refine the basis, $m^{\prime}$ new base points have to be added to the existing ones. We take the union set of all sampling points according to all basis functions which are identified to be refined by the above algorithm and call this union the picking set. For choosing the base points for the additional basis functions, we observe that sampling points with a large distance to the base points $q_{k}$ have a large statistical weight due to the structure of $U_{k}$ and the weight $\gamma$. Consequently, the selection of the additional base points out of the picking set is then done by the picking algorithm in Section III C: New base points are iteratively added having maximal minimal distance to all base points selected so far-including the initial base points. After adding the predefined number of new base points, the scalar parameters $\alpha_{k}, k=m+1, \ldots, m+m^{\prime}$ are again determined according to the smallest distance (only for the added basis functions $\alpha_{j}=\sigma / d_{\text {min }}^{2}$ ). Thus, adding new basis functions only affects the normalization function $\gamma$ and has no impact on the already sampled restraint potentials $U_{k}$. The only change is that the computations of $P$ and $M$ in Equations (11) and (12) have to be applied with regard to the new normalization function $\gamma$.

Since the distances between the base points are assumingly decreasing in each refinement step, $\alpha$ will increase. New simulations according to the restraints $U_{k}, k=m+1, \ldots$, $m+m^{\prime}$ are performed. In order to assure optimal acceptance ratios, the step length of the MC sampling method is necessary. Usually, the step length will decrease leading to less "exploration" of the conformational space within the predefined number of simulated points. In this way the adding of new base points is a real refinement.

\section{F. Exploring additional transitions}

For performing the described meshless algorithm and the refinements, it is not mandatory that there exist one simulated trajectory which covers all parts of the state space $\mathbb{X}$. Even the initial discretization can be based on unconnected samplings of different parts of the conformational space. The advantage of a meshless approach with global radial basis functions (instead of sets) is the assured construction of dense matrices $\tilde{P}$ and $\tilde{M}$. The "transition matrix" $\tilde{P}$ is dense and also accounts for $d$-proximity of the base points (cp. (7)). More precisely, the computed membership functions $\tilde{\chi}$ account for transitions where they occur and for $d$-proximity where transitions do not occur. The discrimination between dynamical and geometrical proximity is coded in the difference between $\tilde{M}$ and $\tilde{P}$, because $\tilde{M}$ only includes the pure proximity information. The extension based stopping criterion in Section III C probably leads us to resolve only the main transitions between the metastable states in the beginning. However, in the refinement procedure later on it is likely to find additional transitions between the identified conformations. The reason is the following. The added base points will be concentrated in those regions of the state space $\mathbb{X}$ where the transitions between the conformations occur. These regions are related to level sets of $\tilde{\chi}_{i} \approx 0.5$. If the refinement is performed "completely along" these level sets, then all transitions between the corresponding conformations are identified in the end. In the illustrative example we will show that the refinement procedure identifies a transition region between two conformations which was not explored in the beginning.

\section{NUMERICAL EXPERIMENTS}

\section{A. Illustrative example}

As an illustrative example the following potential energy function is analyzed:

$$
\begin{aligned}
V(x)= & 3 \exp \left(-x_{1}^{2}-\left(x_{2}-\frac{1}{3}\right)^{2}\right) \\
& -3 \exp \left(-x_{1}^{2}-\left(x_{2}-\frac{5}{3}\right)^{2}\right) \\
& -5 \exp \left(-\left(x_{1}-1\right)^{2}-x_{2}^{2}\right) \\
& -5 \exp \left(-\left(x_{1}+1\right)^{2}-x_{2}^{2}\right) \\
& +0.2 x_{1}^{4}+0.2\left(x_{2}-\frac{1}{3}\right)^{4} .
\end{aligned}
$$

Contour lines of the potential energy function are plotted in Fig. 1. In the numerical experiments the inverse temperature is $\beta=4$, which leads to very rare transitions between the three metastable regions of the potential energy function. On average, only every 100 000th step of the selected Langevin dynamics overcomes energetic barriers and is able to leave one of the major metastable regions. Long-term trajectories are, thus, inefficient in order to sample transition probabilities between the three wells, because the transitions occur rarely. There exists an eigenvalue solution based on the infinitesimal generator of that potential (Eq. (5.3) in Ref. 22). From this solution we get that $\lambda_{2} \approx 0.99998$ and $\lambda_{3} \approx 0.997$.

\section{Two different settings}

In this first example, we investigate the insertion of new basis functions as described in Section III E. More precisely, by setting $\alpha_{j}=\sigma / d_{\min }$ and using different values for $\sigma$, we control the influence of new basis functions onto the shape of all basis functions. By setting first $\sigma=1$, the shape parameter $\alpha$ increases moderately, such that a very deep refinement of the state space is realized, see Figs. 2(a) and 2(c). Second, we chose $\sigma=3$ such that $\alpha$ increases rapidly, and only a few refinement steps are performed. Below, the two 


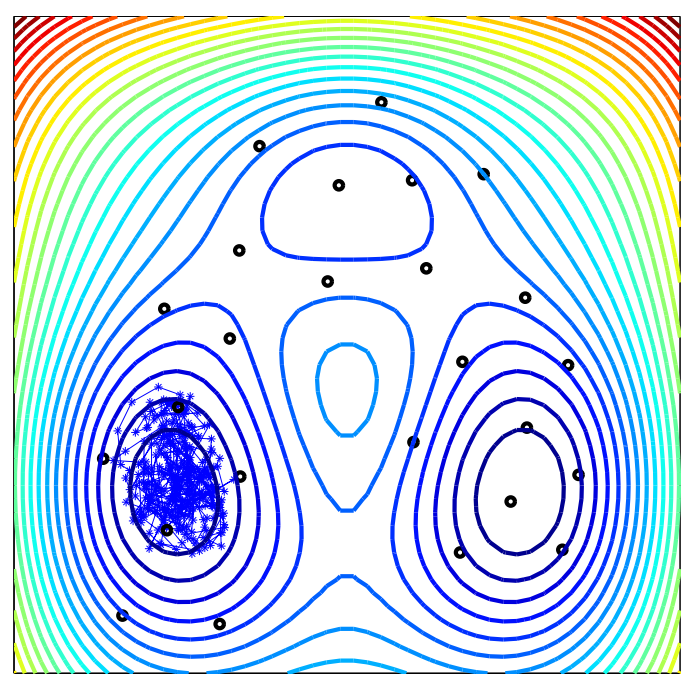

FIG. 1. Contour lines of a three-well potential with two symmetric metastabilities (on the left and on the right) and one less metastable region (top). The blue trajectory shows an initial sampling of the state space $\mathbb{X}$ starting in the left metastable region. 500 steps of a discretized Langevin dynamics have been computed. This number is too low to expect a transition into another metastable region. However, by the restricted samplings, one transition path from the left (via the top) to the right metastable region is detected. The black circles correspond to the initial nodes.

choices of $\sigma$ are discussed based on the results of the rough discretization.

\section{Initial discretization for $\sigma=1$}

The initial discretization of the state space $\mathbb{X}$ has been done according to the above algorithm in Sec. III C. For an initial sampling, we start 500 steps of a diffusion process as described in Eq. (3) in the left minimum (cf. Fig. 1). In the next step, 25 initial base points are selected out of this initial sampling. For each of the corresponding basis functions, we compute the restraint subsampling points according to the restraint potential $U_{k}, k=1, \cdots, 25$. We then employ the picking algorithm (Sec. III C) and subsample the new basis functions. Repeating this procedure enables us to cover three minima with an initial discretization. This corresponds to the black points in Fig. 1.

\section{Refinement for $\sigma=1$}

If we apply the refinement procedure in Sec. III D for determining the basis functions that have to be refined and if we interpret the potential as a 2-metastabilities-situation, then mainly the region which directly connects the two deep minima is refined, see Fig. 2(a). In this region the membership functions have the largest gradients and need to be represented by a finer "grid" of meshless basis functions. If we plot circles with the $r_{k}$-values as radii and base points as centers in conformational space (Fig. 2(b)), we can directly see that in this region the mandatory subspace argument for the wellconditioned computation of $\lambda_{i}$ via $\lambda_{i}=\lambda_{P, i} / \lambda_{M, i}$ is violated at most.

\section{Results for $\sigma=1$}

In the above example, the potential energy function is regarded as a 2-metastabilities-situation with a direct transition between the two deep minima and a (lower minimum) transition region at the top. The refinement procedure stopped, when $\alpha>60$. The computation of the second largest eigenvalue according to $\lambda_{i}=\lambda_{P, i} / \lambda_{M, i}$ yields $\lambda_{2}=0.99996$. Both transition pathways between the main metastabilities have been detected. However, the potential energy function can be seen as a 3-metastabilities-situation too. In that case we aim at computing 3 generalized eigenvectors instead of 2 . Let us rephrase that condition (14) is fundamental for a wellconditioned computation of leading eigenvalues of $T$. For instance, if we would try to compute the third eigenvalue based only on the refinement in Fig. 2(a), then the approximation yields $\lambda_{3}=1.0237$ which is obviously wrong, because $T$ cannot have eigenvalues greater than 1 . Thus, the target invariant subspace condition of the refinement procedure is mandatory.

If we apply the refinement procedure for 3 eigenvectors, then the refinement will not only take place in the region where the two main metastable regions meet each other but also takes place inside the small top basin. This can be seen in Fig. 2(c). The resulting leading eigenvalues are $\lambda_{2}=0.99994$ and $\lambda_{3}=0.99695$.

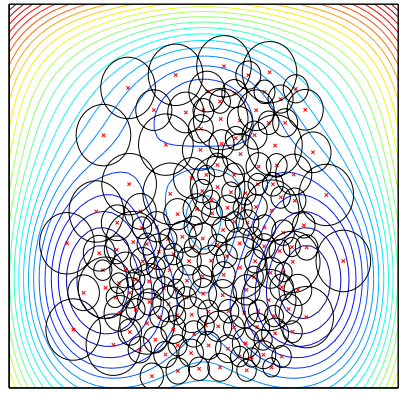

(a)discretization for $\sigma=1$, clusters $=2$

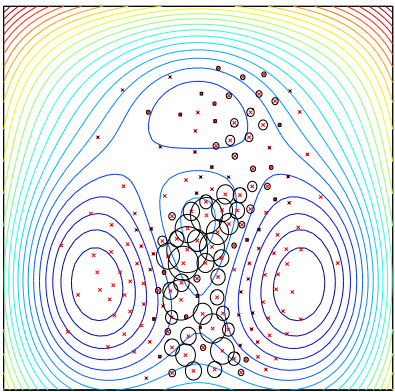

(b)error indicator for $\sigma=1$, clusters $=2$

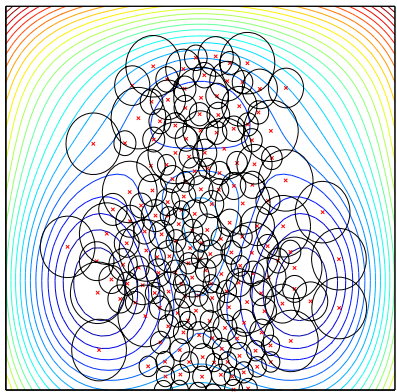

(c) discretization for $\sigma=1$, clusters $=3$

FIG. 2. (a): In this plot we try to identify 2 metastabilities $n=2$. Mainly the direct transition region between the deep minima is refined. The red crosses represent the selected nodes, while the black circles represent the "size" or variance of the penalty potentials. The smaller the circles, the higher $\alpha$. (b): The error evaluation for the 2-metastabilities-situation. Bigger black circles have higher error values $r_{k}$. Clearly, the subspace condition is mostly violated in the direct transition region between the two deep minima. (c): If the refinement procedure is based on 2 metastabilities, then mainly the direct transition region between the deep minima is refined. If the refinement, however, is based on 3 metastabilities, then the top region of the state space $\mathbb{X}$ is refined, too. This is shown here. 


\section{Results for $\sigma=3$}

Using a higher value for $\sigma$ leads to a faster increase of $\alpha$. In the initial phase of the algorithm, a higher value of $\alpha$ leads to an exploration of regions with a higher energy. Thus, both transition pathways between the main metastabilities are already included in the initial discretization. The final discretization is already found after two refinement steps, see Fig. 3. Only near the direct transition region between the two main metastabilities additional base points were located. The resulting eigenvalues of the approximated Galerkin projection are $\lambda_{2}=0.99991$ and $\lambda_{3}=0.99733$. This is already a very good approximation compared to the very fine discretizations. In the numerical experiments, the second eigenvalue mainly increases during the refinement, whereas, the third eigenvalue decreases. There is a reason for this. On the one hand, there is the (Rayleigh's) variational principle. ${ }^{23}$ The better the discretization can resolve the leading eigenfunctions, the higher the eigenvalues are of the corresponding Galerkin projection of the transfer operator. This could explain why $\lambda_{2}$ increases with a finer discretization. On the other hand, the transfer operator is not analytically given, it is represented by a sampling procedure. This means, as long as not all transition pathways are found, the processes seem to be "more metastable" as they are. This could explain that a certain level of discretization has to be reached in order to account for all possible transition pathways in the system. This means that eigenvalues can decrease with a finer discretization, seemingly contradicting the variational principle.

\section{B. Molecular example}

The second example is a nine-dimensional molecular system. In this system 3 argon atoms (in three dimensional space) are interacting with each other. The interaction potential is a Lennard-Jones potential

$$
V_{L J}(r)=\epsilon\left(\left(\frac{r_{0}}{r}\right)^{12}-2\left(\frac{r_{0}}{r}\right)^{6}\right)
$$

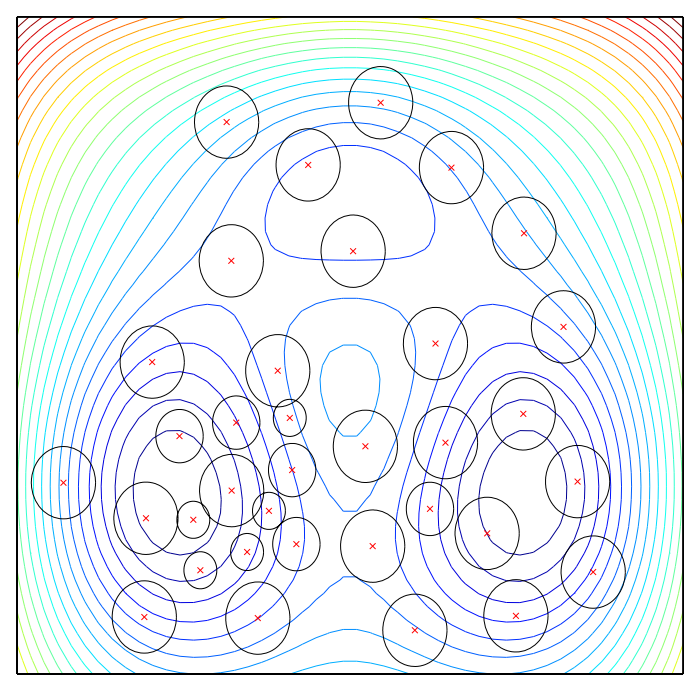

FIG. 3. If the parameter $\sigma$ is high, then $\alpha$ increases rapidly. The algorithm stops after only a few refinement steps of the discretization. The meshless basis functions are indicated in this plot. A fast convergence could lead to unrevealed transition regions. However, the probability to find different transition pathways in the initial discretization phase is high for $\sigma=3$. which depends on the pairwise distance $r$ between the argon atoms. In our example the LJ-energy is $\epsilon=94.87[\mathrm{~kJ} / \mathrm{mol}]$ and the optimal distance between the argon atoms is $r_{0}=3.74 \AA$. In addition to the LJ-potential, there is a quadratic penalty potential to restrict each atom coordinate to be in the interval [-2 $\AA, 2 \AA]$. The inverse temperature has been set to $\beta=0.1$ $\mathrm{mol} / \mathrm{kJ}$ in order to have rare transition events. As in the above example, we set $\sigma=3$ for the adaptive calculation of the parameter $\alpha$ of the modified potential. We set the trial step size to be $0.03 \AA$ for the Metropolis Hastings sampling (MC) of the inner products. This assures an acceptance ratio of about $80 \%$ during our simulations. For the refinement we combine the invariant subspace condition of Sec. III D with a standard Gelman-Rubin criterion. The samplings should at least converge. This is reached, if the Gelman-Rubin-indicator is less than 1.3.

We start our simulation with a conformation, where all pair-wise distances between the argon atoms are optimal, i.e., $r=r_{0}$. The transfer of the molecular process is given by a diffusion process where the drift is based on the gradient of the LJ-potential, see Eq. (3).

This system has four different metastable conformations, see Fig. 4. The first one "1-2-3" is the main conformation, where all distances between the atoms are nearly $r_{0}$. In the other three conformations one atom is unbound and two atoms form a pair; these symmetric conformations can be denoted by "1-2 3, 12-3, 1-3 2." Because of this, we refer to the main conformation as the doubly bound one and call the other three the singly bound conformations.

The given molecular example has three difficulties. First, the starting point of the simulation 1-2-3 has a very low potential energy such that the transition from that conformation to another conformation is very unlikely. Second, the transitions between the conformations of the system are not only driven by the potential energy term, they are also driven by entropic contributions, because the unbound atom has to "find" its binding partners. Thus, there is no saddle point in the potential energy landscape. And third, the

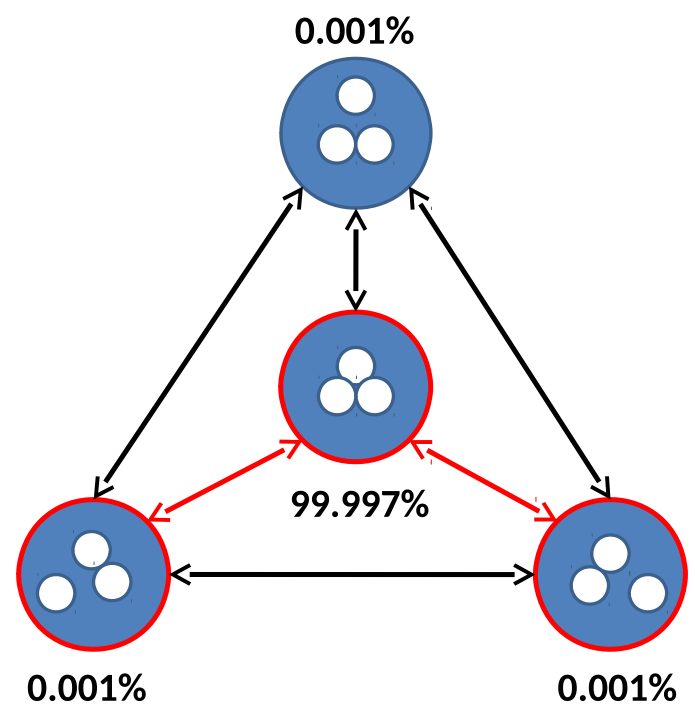

FIG. 4. Statistical weights of the conformations of argon. The red colored circles and arrows are the conformations and transitions that have been found in the initial discretization, i.e., the first row in Table I. 
TABLE I. Showing the refinement steps of the procedure explained in Section III D. Initially, we started the picking procedure with 37500 steps ( 3 iterations of the picking algorithm). The column \# steps corresponds to the number of restraint sampling points. The number of conformations (\# clusters) has been determined with the minChi criterion ${ }^{25}$ and increases during the procedure. The angle (column "subspace") between the subspaces of the eigenvectors $\bar{u}_{P}$ and $M \bar{u}_{P}$ decreases within the refinement steps, whenever the number of clusters is unchanged. This shows that the idea of the refinement indicator applies. Furthermore, we provide the number of additionally picked base points (\# base points), the number of propagated sampling points (\# propagated), and the leading eigenvalues of the discretized transfer operator.

\begin{tabular}{lcccccc}
\hline \hline Refinement level & \# steps & \# propagated & \# base points & Subspace & $\lambda_{P, 2}, \lambda_{P, 3}, \lambda_{P, 4}$ & \# cluster \\
\hline 0 & 37500 & 7500 & 25 & 0.031 & $0.78,0.77,0.68$ & 3 \\
1 & 21000 & 4200 & 14 & 0.029 & $0.78,0.77,0.74$ & 3 \\
2 & 18000 & 3600 & 12 & 0.0602 & $0.78,0.78,0.74$ & 4 \\
3 & 21000 & 4200 & 14 & 0.0394 & $0.79,0.78,0.74$ & 4 \\
\hline Total & 135000 & 19500 & 65 & & & \\
\hline \hline
\end{tabular}

three conformations "1-2 3, 1 2-3, 1-3 2" are equivalent. Thus, the corresponding transition probabilities should be equal.

In the initial discretization phase, two singly bound conformations are already covered. Furthermore, the transitions between these conformations and the doubly bound conformation are revealed. Analyzing the transition matrix after that phase reveals only 2 further eigenvalues close to $\lambda_{1}=1$ (the system should have three). The refinement of the conformational space mainly takes part in the entropic region. The transitions between the singly bound states are discovered at a later stage of the refinement. In Table I the results of the initial level and the three refinement levels are shown. For measuring the similarity between the subspaces spanned by $\bar{u}_{P}$ and $M \bar{u}_{P}$, we computed the angle between the subspaces. ${ }^{24}$

After four refinement steps with 65 base points in total, the analysis of the transition matrix reveals all four conformations, i.e., all transitions indicated in Fig. 4.

Although the discretization is far from being symmetric, the calculated transition probabilities between the conformations are symmetric: The conditional transition probabilities from the minor conformations into the main conformation are in the range of $7 \%-8 \%$. Thus, the maximal absolute deviation from the symmetry is $1 \%$. The eigenvalues $\lambda_{2}, \lambda_{3}$, and $\lambda_{4}$ of $M$ are near 0.84 , while the corresponding eigenvalues of $P$ are near 0.77 . This indicates a relatively good mixing and a good overlap between the meshless basis functions. Although in the meshless approach the eigenvalues are low, the computation of the eigenvalues of $\tilde{M}^{-1} \tilde{P}$ and of the statistical weights reveals that the main conformation covers about 99.997\% of the conformational space, and transitions from that doubly bound conformation into one of the singly bound ones on average only take place every 30000 th time step, if an unrestricted sampling of the diffusion dynamics (3) is applied instead of our meshless discretization approach. In total, we sampled 154500 steps for the whole procedure and figured out the statistical weights and all transition probabilities to correct symmetry.

For comparison, we performed a straightforward simulation without any restraints of the dynamics with 300000 steps (same stepsize as above). An analysis of the trajectory with PCCA+ classifies the atomic distance of $4 \AA$ to be "unbound," whereas, in the meshless approach it was $7 \AA$. The reason for the difference is that the farthest distance between the atoms during the simulation has only been $5 \AA$. This explains also why the main conformation had a lower statistical weight in the straightforward simulation: 299840 steps were found in the main conformation, which are $99.94 \%$ of all steps. In total, the trajectory changed 4 times between the conformations, and only 2 of the 3 unbound conformations were visited. A straight simulation giving the correct symmetry and reaching the conformations where the atoms have a distance of more than $7 \AA$ seems computationally extremely demanding.

\section{CONCLUSION}

In this article, we presented a set-free approach to MSM building utilizing smooth overlapping ansatz functions instead of sets. In addition, an adaptive refinement procedure of this kind of meshless discretization was proposed that allows to improve the quality of the model while exploring state space while in parallel improving the approximation quality of the MSM by inserting new ansatz functions into the discretization.

Furthermore, the choice of Gaussian-like ansatz functions makes it easy to implement the Galerkin projections underlying the discretization by means of existing sampling software via quadratic penalty potentials. We combined this easily available restraint sampling procedure with a reweighting strategy such that the ansatz functions are approximate indicator functions of sets such that the popular interpretation of the resulting MSM in terms of transition probabilities can again be used.

A generalized eigenvalue problem has to be solved for the resulting non-orthogonal Galerkin projection. In order to avoid ill-conditioned eigenvalue problems, we proposed to adaptively refine the ansatz space such that the eigenvectors of the "transition matrix" $P$ are also invariant with regard to the "mass matrix" $M$. It turned out that this approach explores the transition regions of the conformational space, and can reveal conformations of the molecular system which were undiscovered by the initial sampling. 


\section{ACKNOWLEDGMENTS}

This research has been partially funded by Deutsche Forschungsgemeinschaft (DFG) through grant CRC 1114 "Scaling Cascades in Complex Systems," Project (A05) "Probing Scales in Equilibrated Systems by Optimal Nonequilibrium Forcing."

${ }^{1}$ M. Weber, Ph.D. thesis, Free University Berlin, Berlin, 2006.

${ }^{2}$ C. Schütte, A. Fischer, W. Huisinga, and P. Deuflhard, J. Comput. Phys. 151, 146 (1999).

${ }^{3}$ C. Schütte and M. Sarich, Metastability and Markov State Models in Molecular Dynamics: Modeling, Analysis, Algorithmic Approaches, Courant Lecture Notes Vol. 24 (American Mathematical Society, 2013), URL http://publications.mi.fu-berlin.de/1304/.

${ }^{4} \mathrm{C}$. Schütte and W. Huisinga, in Handbook of Numerical Analysis (Elsevier, 2003), pp. 699-744.

${ }^{5}$ An Introduction to Markov State Models and Their Application to Long Timescale Molecular Simulation, Advances in Experimental Medicine and Biology, edited by G. R. Bowman, V. S. Pande, and F. Noé (Springer, 2014), Vol. 797, URL: http://publications.mi.fu-berlin.de/1360/.

${ }^{6}$ F. Nüske, B. G. Keller, G. Pérez-Hernández, A. Mey, and F. Noé, J. Chem. Theory Comput. 10, 1739 (2014).

${ }^{7}$ G. Perez-Hernandez, F. Paul, T. Giorgino, G. de Fabritiis, and F. Noe, J. Chem. Phys. 139, 015102 (2013).

${ }^{8}$ N. Djurdjevac, M. Sarich, and C. Schütte, Multiscale Model. Simul. 10, 61 (2012).

${ }^{9}$ C. Schütte, F. Noé, J. Lu, M. Sarich, and E. Vanden-Eijnden, J. Chem. Phys. 134, 204105 (2011).

${ }^{10}$ E. Vanden-Eijnden and M. Venturoli, J. Chem. Phys. 130, 194101 (2009).
${ }^{11}$ M. Sarich, F. Noé, and C. Schütte, Multiscale Model. Simul. 8, 1154 (2010).

${ }^{12}$ C. Schütte and M. Sarich, Eur. Phys. J.: Spec. Top. 224, 2445 (2015).

${ }^{13}$ P. Deuflhard and M. Weber, Linear Algebra Appl. 398, 161 (2005).

${ }^{14}$ K. Fackeldey, S. Röblitz, O. Scharkoi, and M. Weber, in Particle Methods II, Fundamentals and Applications, edited by E. Onate and D. Owen (International Center for Numerical Methods in Engineering, Barcelona, Spain, 2011), pp. 899-909.

${ }^{15}$ V. Durmaz, Ph.D. thesis, Free University Berlin, 2016.

${ }^{16}$ B. de Groot, D. van Aalten, R. Scheek, A. Amadei, G. Vriend, and H. Berendsen, Proteins 29, 240 (1997).

${ }^{17}$ F. Wende, F. Cordes, and T. Steinke, in Proceedings of the 2012 Symposium on Application Accelerators in High Performance Computing (SAAHPC) (IEEE, Washington, DC, USA, 2012), pp. 74-83.

${ }^{18}$ R. Battiti and G. Tecchiolli, ORSA J. Comput. 6(2), 126-140 (1994).

${ }^{19}$ J. J. Moré and Z. Wu, Large-Scale Optimization with Applications (Springer, 1997), pp. 99-121.

${ }^{20}$ A. Gelman and D. Rubin, Stat. Sci. 7, 457 (1992).

${ }^{21}$ A. Bujotzek, O. Schütt, A. Nielsen, K. Fackeldey, and M. Weber, J. Math. Chem. 52, 781 (2014)

${ }^{22} \mathrm{M}$. Weber, "A subspace approach to molecular Markov state models via a new infinitesimal generator," Habilitation thesis, Fachbereich Mathematik und Informatik, Freie Universität Berlin, 2011.

${ }^{23}$ R. Horn and C. Johnson, Matrix Analysis (Cambridge University Press, 2013).

${ }^{24}$ G. H. Golub and C. V. Loan, in Matrix Computation (John Hopkins University Press, 2013), Chap. 6.4.3.

${ }^{25} \mathrm{M}$. Weber, W. Rungsarityotin, and A. Schliep, "From data and information analysis to knowledge engineering," in 29th Annual Conference of the German Classification Society in Studies in Classification, Data Analysis, and Knowledge, 9-11 March 2005 (Springer, Heidelberg, 2006), pp. 103-110. 